\section{Organic Photodiodes Deposited on Newspaper}

Organic semiconductors have received significant interest recently and are under serious consideration for use in flexible electronics on a wide variety of substrates. In particular, organic photodiodes have seen rapid improvements in properties and performance. In a recent study, B. Lamprecht and co-workers at Günther Leising's Institute of Nanostructured Material and Photonics in Weiz, Austria, a division of Joanneum Research, have demonstrated the fabrication and characterization of organic photodiodes deposited on ordinary newspaper sheets. They reported their results in the April issue of Physica Status Solidi A (p. R50; DOI: 10.1002/pssa.200510010).

The paper samples, taken from the German newspaper Die Zeit, were mounted on glass substrates during the fabrication process. The newspaper substrates were coated with a parylene $C$ barrier layer (5-10 $\mu \mathrm{m}$ thick), which is an effective chemical and moisture barrier. Since the newspaper surface is rough, and the parylene coating does not provide a smoothing effect, an ORMOCER coating, which is a new class of silicate-based inorganicorganic hybrid polymer materials, was applied. The ORMOCER coating, 100 $\mu \mathrm{m}$ thick, provided surface planarization and was inert to subsequent depositions of metal and organic layers. The rms surface roughness at this point was less than $3 \mathrm{~nm}$. The organic charge-generating layers were then deposited, sandwiched between a 55-nm-thick gold bottom electrode and a 25-nm-thick semitransparent silver top electrode. The fabricated organic photodiode, similar to the well-understood Tang-type $p n$-heterojunction device, consisted of a $p$-type conducting copper phthalocyanine ( $\mathrm{CuPc}$ ) and an $n$-type perylene tetracarboxylic bisbenzimidazole. The organic and metal layers were deposited using room-temperature, high-vacuum sublimation. The fabricated devices were removed from the glass substrates prior to characterization.

The current-voltage characteristics of the devices were measured in the dark and under illumination using a halogen lamp, with white light passing through the semitransparent Ag electrode. In the dark, the devices showed a well-pronounced rectification ratio of about $10^{4}$ at $\pm 1 \mathrm{~V}$. On illumination, the devices yielded a photocurrent exceeding the dark current by about six orders of magnitude when operated near a zero bias condition. No hysteresis effect was observed. The photodiodes yielded very good diode behavior. Also, in order to determine the spectral re- sponse of the device, measurements of spectrally resolved external quantum efficiency were carried out using a tungsten halogen lamp and a monochromator. The devices yielded a spectral response covering the complete ultraviolet and visible light range. The researchers said that although newspaper was used as an example, from a wider perspective, this study demonstrated that organic photodiode devices may likely be fabricated on almost any user-defined substrate.

GOPAL RAO

\section{Al-Based Tunnel Junctions Form Solid-State Refrigerator Suitable for Sub-Kelvin Applications}

Many cutting-edge analytical and astronomical devices require cryogenic operating temperatures of $\sim 100 \mathrm{mK}$. Current methods for chilling to the $0.1 \mathrm{~K}$ range, such as adiabatic demagnetization refrigerators (ADR), can be costly and impractical. As an alternative, A.M. Clark and N.A. Miller of the National Institute of Standards and Technology (NIST), along with co-workers from NIST and the University of Notre Dame, have demonstrated a solid-state refrigerator based on normal metal-insulator-superconductor (NIS) junctions composed of $\mathrm{Al}$ and $\mathrm{Al}-\mathrm{Mn}$ electrodes. The refrigerator has limited cooling capacity, but is suitable for critical applications in which a primary refrigerator or liquid cryogen cools a device to subkelvin temperatures, and the solid-state refrigerator provides a secondary cooling source to pull a device below a critical temperature. As reported in the April 25 issue of Applied Physics Letters (173508; DOI $10.1063 / 1.1914966)$, the devices can effectively cool large-area $\mathrm{Si}_{3} \mathrm{~N}_{4}$ membranes as well as electrically isolated bulk payloads placed on the membrane.

The NIS refrigerators (see Figure 1) are

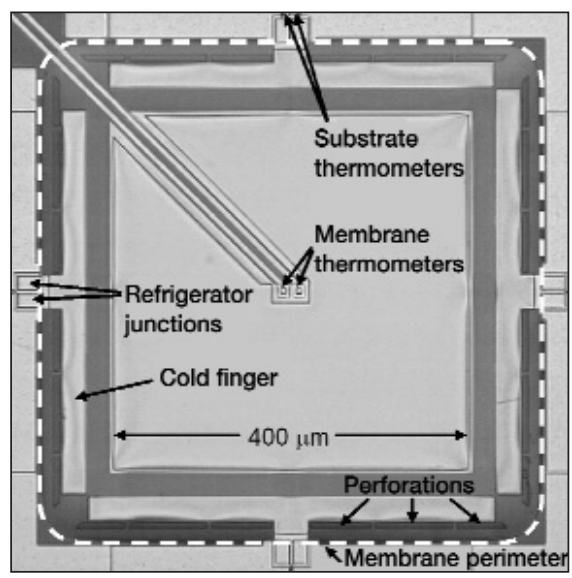

Figure 1. Optical micrograph of metalinsulator-superconductor refrigerator. fabricated using typical thin-film lithography processes, making them easy to integrate with other solid-state devices. The refrigerators are designed with pure $\mathrm{Al}$ as the superconductor, Al doped with $\mathrm{Mn}$ as the normal metal, and an Al-Mn oxide as the insulator. Four pairs of $25 \mu \mathrm{m} \times 15 \mu \mathrm{m}$ NIS junctions surround a $450 \mu \mathrm{m} \times 450 \mu \mathrm{m}$ $\mathrm{Si}_{3} \mathrm{~N}_{4}$ membrane formed by deep reactive ion etching. Al-Mn cold fingers extend from the refrigerator junctions onto the edges of the suspended membrane, and perforations isolate the fingers and membrane from the substrate. The fingers and membrane are coated with separate Au films to enhance thermal conductivity. Additional NIS junctions $(5 \mu \mathrm{m} \times 5 \mu \mathrm{m})$ were incorporated as temperature sensors. When the refrigerator junctions are under bias, the hottest (highest energy) electrons tunnel from the normal metal fingers away into the superconducting metal. The very low phonon thermal conductance (compared to electron or electron-phonon) allows the entire membrane to be cooled by the cold fingers.

The researchers used an ADR to chill the devices for calibration and characterization. At an optimal applied bias of $0.91 \Delta / e$, where $\Delta=189 \mu \mathrm{eV}$ is the superconducting bandgap energy, the devices were able to reduce the membrane temperature from a starting point of $320 \mathrm{mK}$ to $225 \mathrm{mK}$ and from a starting point of $260 \mathrm{mK}$ to $175 \mathrm{mK}$. The refrigerators were able to cool a $250 \mu \mathrm{m}$ cube of doped Ge placed on the membrane from $320 \mathrm{mK}$ to $\sim 240 \mathrm{mK}$. The cooling power is $40-80 \mathrm{pW}$, which is sufficient to cool five cryogenic photon sensors without raising the temperature of the cold fingers by more than $5 \mathrm{mK}$. The researchers are working to further improve performance by better heat-sinking of the superconductor and developing a normal metal with a higher thermal conductivity than the Al$\mathrm{Mn}$. The researchers also indicated that multistage designs should be easy to fabricate and indicated that a three-stage design should be able to cool objects from an initial temperature of $500 \mathrm{mK}$ to $\sim 10 \mathrm{mK}$.

AMANDA GIERMANN

\section{Gold Nanoshell Bioconjugates Used for Molecular Imaging in Living Cells}

Optical imaging is playing a dramatic role in cancer therapy by aiding early detection, critical to reducing mortality rates. Optical imaging of molecular-specific contrast agents offers real-time in vivo monitoring of abnormalities before pathologic changes occur on the atomic level. In the May 1 issue of Optic Letters (p. 1012), C. Loo, R. Drezek, N. Halas, and co-workers from Rice University described how 
nanoshell bioconjugates can be used to effectively target and image human epidermal growth factor receptor 2 (HER2-a relevant biomarker that is prevalent in human breast carcinoma cells). The researchers chose nanoshells composed of a dielectric silica core surrounded by a thin metallic shell composed of gold whose optical properties can be adjusted by controlling their dimensions. Nanoshells have a strong optical resonance that can be tuned in wavelength across the visible and infrared spectrum, allowing the relative contributions of scattering and absorption at a given wavelength to be tuned by controlling the dimensions of the core and the shell. In this study, nanoshells with a 240-nm-diameter silica core and a 35-nmthick gold shell were used. By utilizing these nanoshells, it is possible to conjugate bio-relevant materials, in this case, proteins that facilitate in vivo imaging.

The researchers attached a linker complex-either anti-HER2 or anti-IgG antibodies-PEG (poly ethylene glycol) - to the nanoshell surface. These conjugated nanoshells where exposed to HER2 expressing SKBr3 cells and studied by dark-field microscopy. A significant increase in optical contrast was observed in the HER2 positive cells targeted with anti-HER2-labeled nanoshells as compared to anti-IgG-labeled nanoshells or cells not exposed to the nanoshell conjugates. The researchers said that nanoshellbased conjugates offer the next generation to in vivo imaging due to their nearinfrared tunability, size flexibility, and systemic control of optical properties.

LARKEN E. EULISS

\section{Hybrid Photoelectrodes for Solar Water Splitting}

Efficient production of hydrogen from water using solar energy is a much-soughtafter research goal that has eluded scientists for many years. The wide-bandgap materials that are used in single-junction electrochemical devices to carry out water splitting processes typically absorb sunlight poorly, resulting in low solar-tohydrogen (STH) conversion efficiencies. Recently, E.L. Miller and colleagues from the Hawaii Natural Energy Institute at the University of Hawaii developed a prototype multijunction photoelectrode proposed to more effectively harness the sun's energy for hydrogen production than has been previously possible.

In the May issue of Electrochemical and Solid-State Letters (p. A247), the researchers reported on the performance characteristics of a prototype hybrid photoelectrode (HPE) device and discussed materials developments that will be nec- essary to improve the STH efficiency. An $\mathrm{HPE}$ is a monolithic thin-film device that integrates photoelectrochemical (PEC) and photovoltaic (PV) components in a multiple-junction arrangement. In such a device, the efficiency of hydrogen production is ideally proportional to the photocurrent in the PEC semiconductor, and thus optimum performance is achieved by maximizing this photocurrent.

The prototype HPE described and tested by the research group consists of an $a$ $\mathrm{SiGe} / a-\mathrm{SiGe}$ tandem structure coated with a sputtered $\mathrm{WO}_{3}$ thin film. This device operated at a hydrogen photocurrent level of $0.5 \mathrm{~mA} / \mathrm{cm}^{2}$ in outdoor sunlight conditions. This corresponds to an STH efficiency of $0.6 \%$. The researchers estimated that peak photocurrents of $0.7 \mathrm{~mA} / \mathrm{cm}^{2}$ could be obtained from this device.

Although the results are promising, these materials clearly do not offer optimal device performance; novel materials must be developed for use as the PEC and PV components. One upgrade suggested by the researchers is the use of "improved" sputtered $\mathrm{WO}_{3}$ coatings. These films offer peak photocurrents of $2.4 \mathrm{~mA} / \mathrm{cm}^{2}$, which represent a threefold increase in peak photocurrent and ideally a corresponding improvement in STH. The researchers predict that when integrated with a newly developed $a-\mathrm{Si} / a-\mathrm{Si}$ tandem structure, an HPE with this improved coating layer would yield STH efficiencies exceeding $2.2 \%$.

The chief limitation of HPE structures at the moment is the maximum photocurrent allowed by the top PEC semiconductor films. Doped $\mathrm{TiO}_{2}$ or $\mathrm{WO}_{3}$ films may offer a solution; these feature photocurrent values as high as $5 \mathrm{~mA} / \mathrm{cm}^{2}$. The researchers also suggest that appropriate combinations of PEC and PV materials may yield STH values in excess of $10 \%$. Clearly, much development is necessary to attain such efficient hydrogen production in HPE devices, but these prototype results point to possible significant improvements in efficiency.

\section{ANDY FRANCIS}

\section{Distribution of Nanoparticles in Photopolymer Controlled Holographically}

Applications for holograms include three-dimensional displays, data storage, and photonics. In recent years, holographic gratings have been fabricated from twocomponent mixtures of organic photopolymers and from polymer-dispersed liquid crystals. The incorporation of materials that extend the range of the refraction index profile will result in more efficient holograms. For example, a system com- posed of organic photopolymers and inorganic nanoparticles, which, unlike organic compounds, display a wide range of refractive indices $(n)$, were recently proposed by researchers from the Department of Electronics Engineering, University of Electro-Communications (UEC), Tokyo, although heretofore, the formation mechanism of such holographic gratings was not clearly understood.

As reported in the April 15 issue of Optics Letters, UEC researchers Y. Tomita and N. Suzuki and K. Chikama from Chemical Research Laboratories, Nissan Chemical Industries, demonstrated holographic control of morphology in nanoparticle-dispersed photopolymers and explained the formation of the holographic gratings in relatively simple terms of the chemical potential $(\mu)$ of non-interacting particles. The researchers combined either $\mathrm{SiO}_{2}$ nanoparticles $(n=1.46$, diameter $=$ $13 \mathrm{~nm})$ or $\mathrm{TiO}_{2}$ nanoparticles $(n=2.55$, diameter $=15 \mathrm{~nm}$ ) at a volume fraction of 0.34 with methacrylate monomers $(n=1.55$ in the liquid and 1.59 in the solid phase at a wavelength of $589 \mathrm{~nm}$ ) and the initiator titanocene to cast films $\sim 50 \mu \mathrm{m}$ thick. Transmission-type holograms were then recorded at a grating spacing of $1 \mu \mathrm{m}$ by exposing the films to two mutually coherent beams at a wavelength of $532 \mathrm{~nm}$. The researchers used transmission electron microscopy to show that the nanoparticles followed the intensity interference fringe pattern at a grating spacing of $1 \mu \mathrm{m}$.

Tomita and his colleagues hypothesized a mutual diffusion model in which monomers polymerize in the bright regions, where their $\mu$ decreases, leading to the diffusion of monomers from dark to bright regions. The photoinsensitive inorganic nanoparticles, on the other hand, are not consumed during polymerization, and their $\mu$ increases in the bright regions, causing them to diffuse to the dark regions. The researchers reasoned that the phase shift $(\phi)$ between the intensity interference fringe pattern and the recorded holograms should be $0^{\circ}$ for the $\mathrm{SiO}_{2}$ hologram, that is, the change in $n(\Delta n)$ is highest in the bright regions, because $n$ for $\mathrm{SiO}_{2}$ is less than $n$ for the polymer. Similarly, $\phi$ for the $\mathrm{TiO}_{2}$ hologram should be $180^{\circ}$, that is, $\Delta n$ is highest in the dark regions, because $n$ for $\mathrm{TiO}_{2}$ is greater than $n$ for the polymer. Both of these predictions were confirmed by the researchers.

Tomita and his colleagues said that their finding of all-optical control of nanoparticle distribution in photopolymers will facilitate the addition of new functionality to holographic applications, such as the fabrication of nonlinear periodic structures and nonlinear photonic 